\title{
The prognostic value of red blood cell distribution width in patients with suspected infection in the emergency department
}

\author{
Jan Willem Uffen ${ }^{1 *}$ (D, Patrick Oomen ${ }^{1}$, Marieke de Regt ${ }^{2}$, Jan Jelrik Oosterheert ${ }^{2}$ and Karin Kaasjager ${ }^{1}$
}

\begin{abstract}
Background: Sepsis is a potential life threatening dysregulated immune response to an infection, which can result in multi-organ failure and death. Unfortunately, good prognostic markers are lacking in patients with suspected infection to identify those at risk. Red blood cell distribution width (RDW) is a common and inexpensive hematologic laboratory measurement associated with adverse prognosis in multiple diseases. The aim of this study was to determine the prognostic value of RDW for mortality and early clinical deterioration in patients with a suspected infection in the emergency department.
\end{abstract}

Methods: In this single center prospective observational cohort study, consecutive patients with suspected infection presenting for internal medicine in the emergency department between September 2016 and March 2018 were included. For prognostic validation of bedside sepsis scores and RDW receiver operating characteristics were generated. Association between RDW and mortality and ICU admission was analyzed univariate and in a multivariate logistic regression model.

Results: 1046 patients were included. In multivariate analyses, RDW was significantly associated with 30-day mortality (OR 1.15, 95\% Cl: 1.04-1.28) and early clinical deterioration (OR 1.09, 95\% Cl: 1.00-1.18). For 30-day mortality RDW had an AUROC of 0.66 (95\% Cl 0.59-0.72). Optimal cut-off value for RDW 2 was $12.95 \%$. For early clinical deterioration RDW had an AUROC of $0.59(95 \% \mathrm{Cl} 0.54-0.63)$ with an optimal cut-off value of $14.48 \%$.

Conclusions: RDW was found to be a significant independent prognostic factor of 30-day mortality and early clinical deterioration in patients with suspected infection.. Therefore it can be a used as an extra marker besides bedside sepsis scores in identifying patients at risk for worse outcome in patients with suspected infection.

Keywords: Infection, Sepsis, Emergency department, Emergency medicine, Internal medicine, Biomarkers

\section{Background}

Sepsis is a clinical syndrome currently defined as a lifethreatening organ dysfunction caused by a dysregulated host immune response to infections [1]. Sepsis has a high incidence, with up to global estimates of 31 million sepsis and 19 million severe sepsis cases, resulting in 5 million deaths annually [2].

\footnotetext{
* Correspondence: J.W.Uffen@umcutrecht.nl

${ }^{1}$ Department of Internal Medicine, division Acute Medicine, University

Medical Centre Utrecht, Heidelberglaan 100, 3584, CX, Utrecht, the

Netherlands

Full list of author information is available at the end of the article
}

Unfortunately, no good diagnostic tool is available for early identification of patients with sepsis and a golden diagnostic standard does not exist [3-6]. In clinical practice, prognostic sepsis scores are often used to identify patients in need of immediate treatment. The Systemic Inflammatory Response Syndrome (SIRS) score, introduced in 1992 and updated in 2001 proved to be insufficiently specific to correctly identify patients most at risk of dying. The prognostic quick Sequential Organ Failure Assessment (qSOFA) score, introduced in 2016 to overcome this problem, lacks sensitivity to identify all patients that are the most at risk of developing sepsis and

(c) The Author(s). 2019 Open Access This article is distributed under the terms of the Creative Commons Attribution 4.0 International License (http://creativecommons.org/licenses/by/4.0/), which permits unrestricted use, distribution, and reproduction in any medium, provided you give appropriate credit to the original author(s) and the source, provide a link to the Creative Commons license, and indicate if changes were made. The Creative Commons Public Domain Dedication waiver (http://creativecommons.org/publicdomain/zero/1.0/) applies to the data made available in this article, unless otherwise stated. 
thus require immediate treatment $[1,7,8]$. The National Early Warning Score (NEWS) or Modified Early Warning Score (MEWS) have been suggested as better performing alternatives in the Emergency Department (ED) $[9,10]$ and use is adopted by many hospitals but they are not part of standardized care yet. Therefore, there is an urgent need for better prognostic markers in sepsis that can be used at bedside.

Red Blood Cell Distribution Width (RDW), a quantitative measure of variability in size of circulating erythrocytes usually determined in a complete blood count (CBC), is an inexpensive and readily available measurement, that may act as a prognostic factor in several diseases. For example, elevated RDW has been associated with adverse prognosis in various non-infectious [11-17] and infectious diseases [18-20], and in patients diagnosed with (severe) sepsis and septic shock [21-26]. But it has also been demonstrated to be of prognostic value in undetermined populations [27]. The exact pathophysiologic mechanism underlying this association is unclear but systemic factors that alter erythrocyte homeostasis such as inflammation and oxidative stress, both essential components of the infection cascade, seem to play an important role [28-36].

Though the independent association with adverse prognosis in various diseases and sepsis has been established, previous research has focused mainly on well-defined populations such as severe sepsis or septic shock, which hampers the generalizability as a prognostic tool in ED populations. Here, we determine the prognostic value of RDW in patients with suspected infection in the emergency department.

\section{Methods}

\section{Study design and setting}

A retrospective analysis of data from the SPACE-cohort (SePsis in the ACutely ill patients in the Emergency department) between September 2016 and March 2018, was performed. Within the SPACE-cohort, all consecutive patients, age $\geq 18$ years, with a suspected infection presenting for internal medicine in the ED of the University Medical Centre Utrecht (UMCU) since September 2016 are prospectively included. The UMCU is a 1042-bed tertiary academic teaching hospital in the Netherlands with approximately 20.500 annual ED visits.

No exclusion criteria were used. Triage for sepsis was performed for all patients presenting for internal medicine in the ED. When sepsis was suspected protocolled care according to a care pathway was initiated. All other patients received protocolled care according to their clinical presentation. The SPACE cohort was reviewed and approved by the Medical Ethical Committee of the UMCU under number 16/594 and registered in the Dutch Trial Register (NTR) under number 6916.

\section{Population and data collection}

The SPACE cohort consists of all consecutive patients who meet the following criteria: $(1) \geq 18$ years or older; (2) presentation at the ED with suspected infection defined by the treating physician in the ED; (3) registration in the ED for the internal medicine department or its subspecialties oncology, rheumatology, immunology, hematology, nephrology, endocrinology, gastro-enterology, geriatrics, infectious disease and vascular medicine. All patients received standard care.

The domain of patients with suspicion of infection by their treating physician was deliberately chosen, as this is the exact group a clinician would like to diagnose or rule out the presence of sepsis. Suspicion of infection was documented for all patients by the attending physician at the ED using an automated record system asking this question followed by the question whether a sepsis was present at the time of presentation or not. When both questions are answered the record system automatically adds the parameters needed to calculate SIRS and qSOFA to the patient record. Furthermore the record system gives a warning to the physician when one or both scores are abnormal.

Independent trained physicians analyzed all patients records on documented suspected infection or sepsis. In absence of documentation, the independent physicians registered both items. Suspicion of infection was considered present, when respectively infection or sepsis was recorded by the treating physician in the ED as diagnosis and/or differential diagnosis in the patient record.

For all patients, demographic parameters such as age and gender and clinical parameters such as temperature, heart rate, respiratory rate, saturation, blood pressure, Glasgow coma scale (GCS), laboratory results including RDW, hospital or intensive care unit (ICU) admission and follow-up data on mortality were automatically collected from electronic medical records. SIRS and qSOFA scores were automatically calculated based on the first recorded data.

Data on comorbidities and immunocompromised status were manually extracted from the patient record system by researchers, using a predefined set of welldescribed definitions. If GCS was not registered, free text notes by the treating physician on the ED were used to derive information on the mental status from.

If data was missing on parameters needed to calculate sepsis scores such as SIRS and qSOFA we chose to score these parameters as normal under the assumption that if patients had abnormal parameters clinicians would have entered these into the electronic medical file.

\section{Laboratory measurements}

Laboratory parameters such as blood chemistry and $\mathrm{CBC}$, including $\mathrm{RDW}$, were performed when patients 
presented in the ED, and the results were obtained within $1 \mathrm{~h}$. RDW is calculated as the standard deviation of erythrocyte volume divided by the mean corpuscular volume (MCV). The reference range of RDW varies between 10.0$16.0 \%$, depending on the used analyzer. In this study, RDW was measured as part of the automated $\mathrm{CBC}$ using the CELL-DYNN Sapphire hematology analyzer (Abbott Diagnostics, Santa Clara, CA, USA). The reference range in the $\mathrm{UMCU}$ is 10.5 to $13.5 \%$.

\section{Outcomes}

The primary outcome of this study was defined as all-cause mortality within 30 days after ED presentation. The secondary outcome was defined as early clinical deterioration defined as death or admission to either ICU or Medium Care Unit (MCU) within 3 days after ED presentation.

\section{Statistical analysis}

Continuous data were expressed as mean \pm standard deviation (SD) if normally distributed or median and interquartile range in the case of non-parametric data. Independent samples t-tests and Mann-Whitney $U$ tests were used to compare parametric and non-parametric continuous variables, respectively. Categorical variables were compared using Chi-Square or Fisher's exact test, depending on cell counts.

The association between RDW and outcomes was studied in a multivariate binary logistic regression model, together with variables with a $p$-value of $<0.2$ in univariate analysis and clinically relevant variables.
For prognostic validation of SIRS, qSOFA, MEWS and RDW receiver operating characteristics (ROC) curves were generated, and the area under receiver operating characteristic (AUROC) was calculated.

Optimal cut-off value of RDW was determined by using Youden's J-statistic in above mentioned ROC curve.

All statistical analyses were performed using SPSS version 25.0 (IBM Corp., Armonk, NY). P-values < 0.05 were considered statistically significant.

\section{Results}

\section{Patient characteristics}

During the study period, 1119 patients were included in the SPACE cohort, of which 1048 (93.5\%) had an RDW measurement at ED presentation and were therefore available for further analysis. In the study cohort 53.8\% $(n=563)$ of patients were male and the median age is 61 years old (IQR 50-72). Table 1 shows the descriptive characteristics of the study population, divided in survivors and non-survivors.

Compared to non-survivors, surviving patients are slightly younger (median age 61.0 versus $68.0(p<$ 0.001)), have a lower Charlson Comorbidity Index (CCI) (median 4.0 versus $7.0, p<0.001$ ) and more often had a lower qSOFA, SIRS score and MEWS at ED presentation (qSOFA $\geq 2: 6.7 \%$ versus $26.2 \%, p<0.001$ ); (SIRS score $\geq$ 2: $61.9 \%$ versus $75.4 \%, p=0.035$ ); (MEWS: 2 versus 3 , $p<0.001)$. Median RDW at ED presentation was $13.42 \%$ (IQR 12.02-14.83). Survivors had a significantly lower RDW (13.38 IQR 12.27-14.99) compared to nonsurvivors (14.74 IQR 13.23-16.71, $p<0.001$ ).

Table 1 Baseline characteristics of the study population according to survival and non-survival at 30-days

\begin{tabular}{|c|c|c|c|c|}
\hline & Total $(n=1046)$ & Non-survivors $(n=61)$ & Survivors $(n=985)$ & $p$-value \\
\hline Gender (male) - no. (\%) & $563(53.8)$ & $37(60.7)$ & $526(53.4)$ & 0.270 \\
\hline Age - yr - median (IQR) & $61.0(50-72)$ & $68.0(61.5-78.0)$ & $61.0(46.0-69.0)$ & $<0.001$ \\
\hline \multicolumn{5}{|l|}{ Comorbidities } \\
\hline Hematologic malignancy - no. (\%) & $178(17.0)$ & $10(16.4)$ & $168(17.1)$ & 0.960 \\
\hline Chronic renal failure $\geq$ stage 3 - no. (\%) & 204(19.5) & $13(22.4)$ & $191(19.4)$ & 0.713 \\
\hline Immunocompromised - no. (\%) & $392(37.5)$ & $15(24.6)$ & $377(38.3)$ & 0.092 \\
\hline Neutropenia due to systemic chemotherapy - no. (\%) & $45(4.3)$ & $1(1.7)$ & $44(4.5)$ & 0.999 \\
\hline $\mathrm{CCl}$ - median (IQR) & $4.0(2-7)$ & $7.0(5-9)$ & $4.0(2-6)$ & $<0.001$ \\
\hline Vasopressor need - no. (\%) & $36(3.4)$ & $8(13.1)$ & $28(2.8)$ & $<0.001$ \\
\hline RDW (\%) median (IQR) & $13.42(12.30-15.11)$ & $14.74(13.23-16.71)$ & $13.38(12.27-14.99)$ & $<0.001$ \\
\hline \multicolumn{5}{|l|}{ Disease severity scores } \\
\hline qSOFA $\geq 2-$ no (\%) & $82(7.8)$ & $16(26.2)$ & $66(6.7)$ & $<0.001$ \\
\hline SIRS score $\geq 2$ - no (\%) & $656(58.6)$ & $46(75.4)$ & $610(61.9)$ & 0.035 \\
\hline MEWS - median (IQR) & $2(1-4)$ & $3(1-5)$ & $2(1-4)$ & $<0.001$ \\
\hline Sepsis according to emergency physician - no. (\%) & $160(15.3)$ & $17(27.9)$ & $143(14.5)$ & 0.005 \\
\hline
\end{tabular}

No number, Yr year, IQR Interquartile range, CCI Charlson Comorbidity Index, qSOFA quick Sequential (Sepsis-related) Organ Failure Assessment, SIRS Systemic Inflammatory Response Syndrome, MEWS Modified Early Warning Score, RDW Red Blood cell Distribution Width 
Table 2 Multivariate logistic regression analysis for 30-day mortality

\begin{tabular}{llll}
\hline & Odds Ratio & $95 \% \mathrm{Cl}$ & $p$-value \\
\hline Age & 1.03 & $1.00-1.05$ & 0.03 \\
CCI & 1.19 & $1.07-1.32$ & 0.00 \\
Immunocompromised & 0.59 & $0.30-1.12$ & 0.11 \\
Vasopressor needs & 2.97 & $1.12-7.91$ & 0.03 \\
qSOFA $\geq 2$ & - & & \\
SIRS score $\geq 2$ & - & & \\
MEWS & 1.22 & $1.08-1.37$ & 0.00 \\
RDW & 1.15 & $1.04-1.28$ & 0.01 \\
\hline
\end{tabular}

CCI Charlson Comorbidity Index, qSOFA quick Sequential (Sepsis-related) Organ Failure Assessment, SIRS Systemic Inflammatory response syndrome, MEWS Modified Early Warning Score, RDW Red Blood cell Distribution Width

\section{Primary outcome}

In total, 61 (5.8\%) patients died within 30 days. For 30day mortality a SIRS score $\geq 2$ had a specificity of 0.38 (95\% CI $0.35-0.41)$ and sensitivity of 0.75 (95\% CI $0.62-0.85)$. qSOFA $\geq 2$ had a specificity of 0.95 (95\% CI $0.94-0.96)$ and a sensitivity of $0.26(0.16-0.39)$. In an univariate logistic regression analysis age, CCI, vasopressor needs, qSOFA $\geq 2$, a SIRS score $\geq 2$, MEWS and RDW were associated with 30-day mortality. Multivariate analysis resulted in age, CCI, vasopressor needs, MEWS and RDW as independent factors associated with 30-day mortality (Table 2). ROC curves for 30-day mortality of RDW, the SIRS score, the qSOFA and the MEWS are shown in Fig. 1 and corresponding AUROCs are shown in Table 3. The optimal cut-off value of RDW was determined at $12.95 \%$.

\section{Secondary outcome}

In this study, $132(12.6 \%)$ patients died within 3 days after ED presentation or were admitted to the ICU/ $\mathrm{MCU}$ as proxy for early clinical deterioration. Compared to patients with no early deterioration they were older (median age 66 versus 60 years, $p<0.001$ ) and more frequently had a SIRS score $\geq 2(81.1 \%$ versus $60.1 \%, p<0.001)$, a qSOFA $\geq 2(30.3 \%$ versus $4.6 \%, p<$ 0.001 ) and a higher MEWS (2 versus $4, p<0.001$ ). Furthermore, patients had higher median RDW-levels (14.04\% (IQR 12.61-15.92) vs. 13.37\% (IQR 12.2614.98), $p=0.001)$. In a multivariate logistic regression model RDW was associated with early clinical deterioration (OR 1.09 (95\% CI 1.00-1.18)). ROC curves for early clinical deterioration of SIRS, qSOFA, MEWS and RDW are shown in Fig. 2 with corresponding AUROCS in Table 3.

Table 4 shows the factors associated with the secondary outcome. Optimal cut-off point for RDW on clinical deterioration was determined at $14.48 \%$.

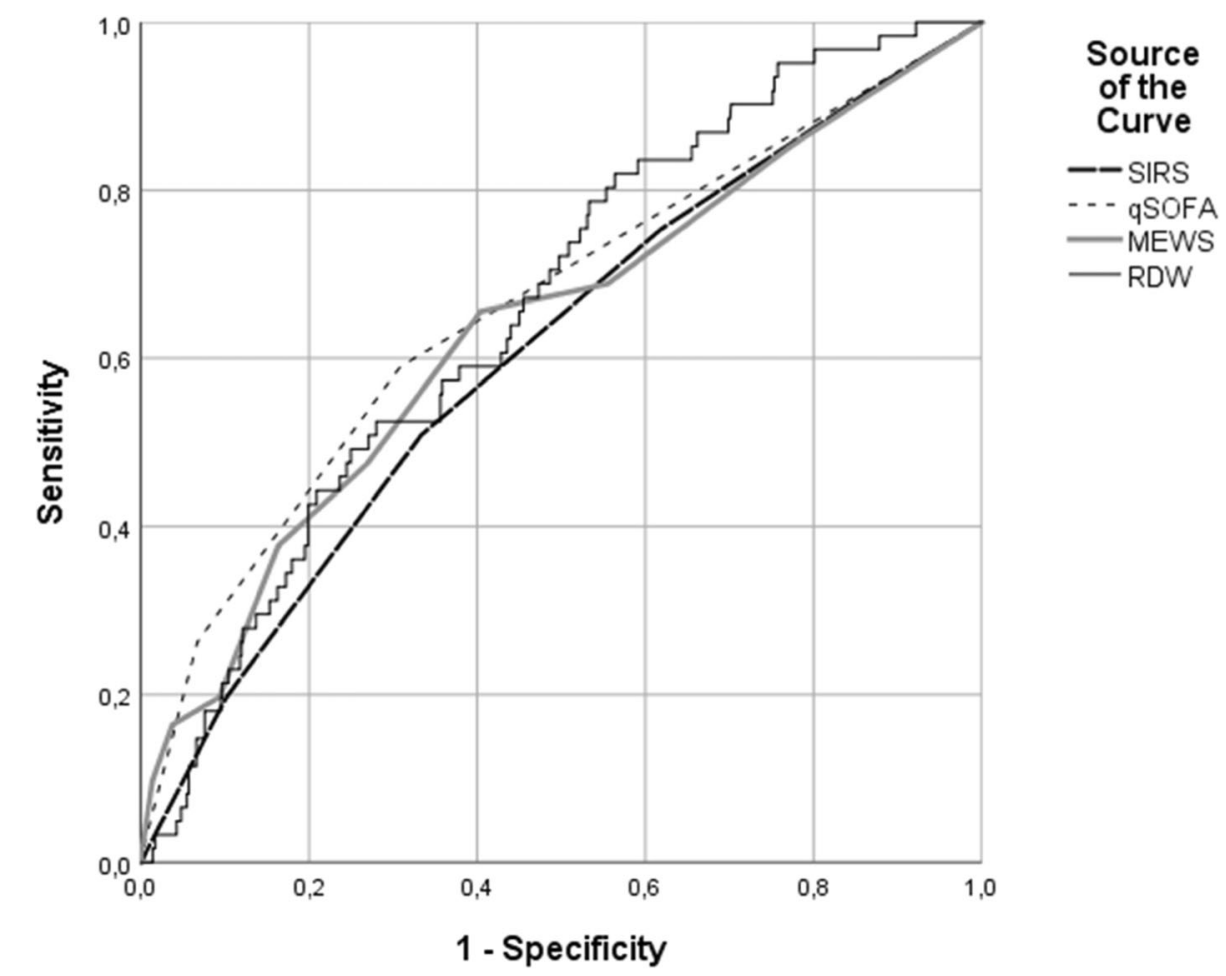

Fig. 1 Receiver operator characteristic (ROC) curve for prediction of 30-day mortality. shown are ROC curves of SIRS, qSOFA, MEWS and RDW for 30-day mortality 
Table 3 AUROCS of RDW, SIRS, qSOFA and MEWS

\begin{tabular}{llllll}
\hline & \multicolumn{2}{l}{ Primary outcome } & & \multicolumn{2}{l}{ Secondary Outcome } \\
\cline { 2 - 3 } & AUROC & $95 \% \mathrm{Cl}$ & & AUROC & $95 \% \mathrm{Cl}$ \\
\hline RDW & 0.66 & $0.59-0.72$ & & 0.59 & $0.54-0.64$ \\
SIRS Score & 0.61 & $0.53-0.68$ & & 0.68 & $0.63-0.72$ \\
qSOFA & 0.66 & $0.59-0.74$ & & 0.76 & $0.71-0.81$ \\
MEWS & 0.64 & $0.58-0.71$ & & 0.74 & $0.69-0.78$ \\
\hline
\end{tabular}

RDW Red cell Distribution Width, SIRS Systemic Inflammatory Response Syndrome, qSOFA quick Sequential (Sepsis-related) Organ Failure Assessment, MEWS Modified Early Warning Score

\section{Discussion}

RDW is a common, inexpensive and relatively fast available laboratory measurement and in ED settings in patients with suspected infection it is independently associated with 30-day mortality and early clinical deterioration. Additional in our multivariate analysis the MEWS was also independently associated with 30-day mortality, as SIRS $\geq 2$ and qSOFA $\geq 2$ were not.

The prognostic power of RDW for 30-day mortality is comparable to that of SIRS, qSOFA and MEWS, but for early clinical deterioration RDW is outperformed by all these bedside scores. Our results are in line with previous studies, that showed an independent prognostic value of RDW in sepsis and comparable AUROCs. However, previous studies investigated more defined homogeneous patient groups (e.g. diagnosed sepsis or septic shock) and more severely ill patients. In patients with established diagnose of severe sepsis or septic shock in the ED, non-survivors had significantly higher RDW-levels [23, 25, 26]. In ICU patients with severe sepsis or septic shock, higher RDW-levels were also observed in non-surviving patients [21, 22, 24]. Our study shows that the independent prognostic value also applies to a more heterogeneous group of patients with suspected infection.

Higher sepsis scores are associated with higher RDWlevels suggesting more sick patients have higher RDWlevels. Even though this suggest an association between RDW and higher scores, RDW still remained an independent predictor for mortality after correction for disease severity.

The present study has several strengths. To our knowledge, this is the first study that examines RDW in a heterogeneous population, in which we did not use any exclusion criteria. The study population consisted of patients who were suspected of infection and were admitted to the ED. This is the exact patient population seen daily in EDs and exactly these patients are most at risk of developing sepsis. Therefore results obtained from this study are directly applicable to the daily practice. Additionally, our study had a fairly large sample size which made for precise results. Furthermore, all-cause

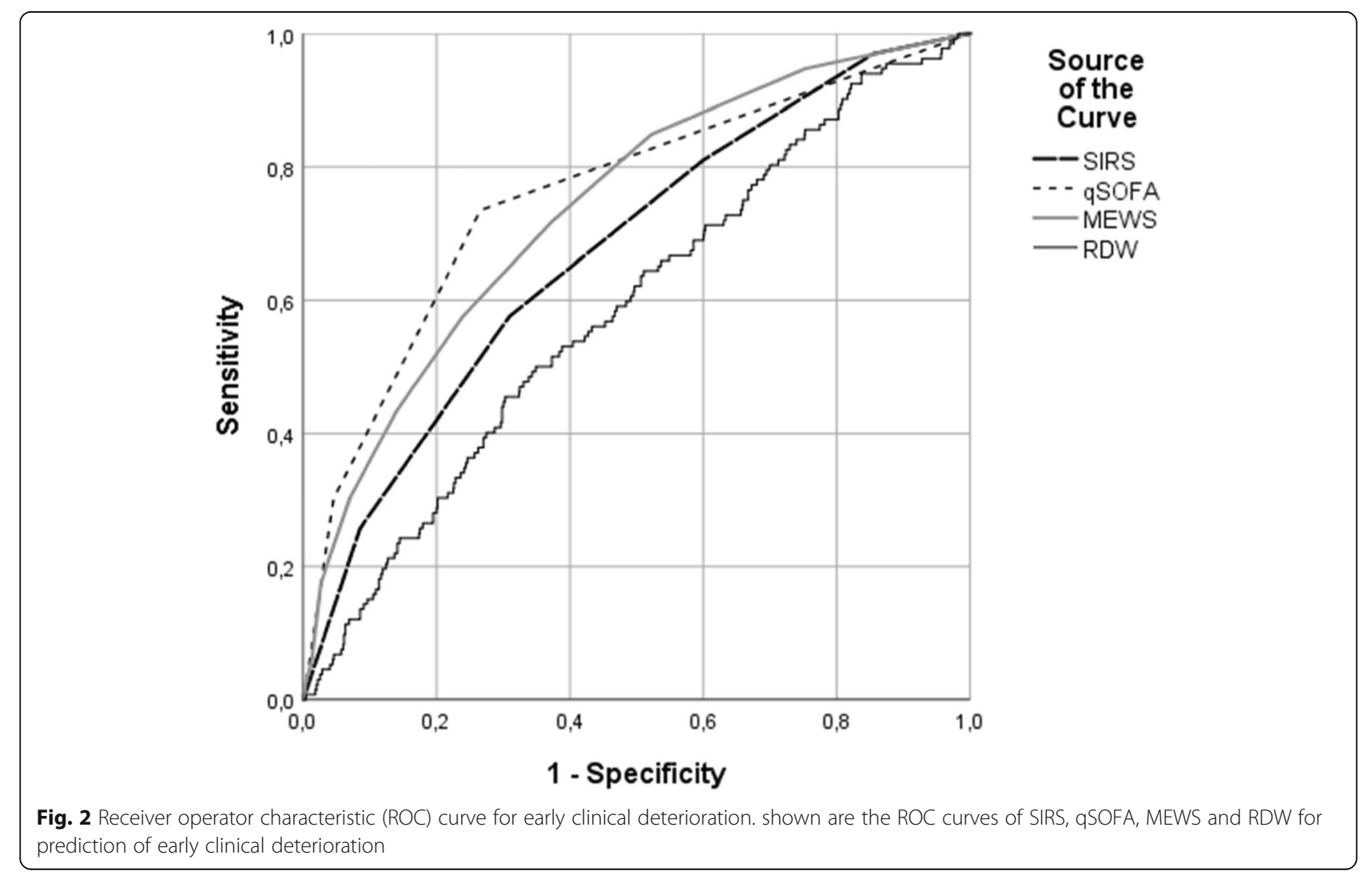


Table 4 Multivariate logistic regression analysis for ICU/MCU admission or death within 3 days

\begin{tabular}{llll}
\hline & Odds Ratio & $95 \% \mathrm{Cl}$ & \\
\hline Gender (F) & & $1.00-1.03$ & 0.03 \\
Age & 1.01 & $0.26-0.96$ & 0.04 \\
Hematologic Malignancy & 0.50 & $0.04-1.5$ & \\
Neutropenia due to systemic chemotherapy & 0.23 & $6.70-39.5$ \\
CCI & & $3.60-10.11$ \\
Vasopressor needs & 16.27 & & 0.23 \\
qSOFA $\geq 2$ & 2.03 & $1.20-1.46$ & 0.00 \\
SIRS $\geq 2$ & - & $1.00-1.18$ & 0.00 \\
MEWS & 1.32 & 1.09 & 0.06 \\
RDW (\%) & 1.00
\end{tabular}

CCI Charlson Comorbidity Index, qSOFA quick Sequential (Sepsis-related) Organ Failure Assessment, SIRS Systemic Inflammatory response syndrome, RDW Red Blood cell Distribution Width

mortality is an unbiased and relevant outcome in observational prognostic studies.

Certain limitations apply to the current study. Firstly, this was a retrospective single center study based on data from electronic medical records, which is a potential source of bias and errors at the time of data recording at the source. Furthermore, due to the heterogeneous population, there is an increased risk that not all confounding factors have been accounted for. Also, being a single-center study and conducted in a large tertiary care institution, results may not be generalizable to other health care institutions.

Although we took conditions that could have influenced RDW-values into account (i.e. hematologic malignancies, chronic renal failure and neutropenia), RDW-values also could have been influenced by nutritional status. This study did not take iron, vitamin B12, folic acid, erythropoietin levels into account. Additionally, blood transfusion records were not available. Though we did not correct for these factors this study shows that RDW-values are applicable for a heterogeneous group of patients and certain comorbidities or conditions should be taken into account when interpreting RDW-values.

Furthermore, a percentage of qSOFA $(2,8 \%)$ and SIRSscores $(25,5 \%)$ could not be determined due to missing data on respiratory rates. We chose to score these as normal and analyzed the at least achieved score results. This under the assumption that if patients would have been tachypnoeic, clinicians would have entered the respiratory rate into the electronic medical file. Missing data in the ED on vital signs is a well-known problem. Especially completing all vital parameters in one single patient. Failure to register vital signs more often when they appear to be normal. Lower triage categories and less sick patients are associated with lesser recording of vital signs [37]. This could have led to underestimation of SIRS scores and to lesser extent qSOFA. To overcome this problem a complete case analysis has been performed which led to no different results.

\section{Conclusion}

RDW, a common and inexpensive laboratory measurement, usually determined in the $\mathrm{CBC}$, was found to be a significant independent prognostic factor of 30-day mortality and early clinical deterioration in patients with suspected infection and has comparable prognostic accuracy compared to clinical sepsis scores as SIRS qSOFA or MEWS in predicting 30-day mortality. Therefore it can be a used as an extra marker besides these bedside scores in identifying patients at risk for worse outcome.

\section{Abbreviations}

AUC: Area under the curve; CBC: Complete blood count; CCl: Charlson comorbidity index; Cl: Confidence interval; ED: Emergency department; ICU: Intensive care unit; MCU: Medium care unit; OR: Odds ratio; qSOFA: Quick Sequential Organ Failure Assessment; RDW: Red blood cell differentiation width; ROC: Receiver operating characteristics; SIRS: Systemic inflammatory response syndrome; SPACE: Sepsis in the acutely ill patient in the emergency room; UMCU: University Medical Centre Utrecht

\section{Acknowledgements}

Values on RDW were collected and provided to this study by the Utrecht Patient Orientated Database (UPOD).

\section{Authors' contributions}

$J U, P O$ and MR jointly conceived the study. PO analyzed data with assistance from $J U$. JU and PO drafted the manuscript. MR, JO and KK commented on the manuscript and provided advice. All authors read and approved the final manuscript.

\section{Funding}

Not applicable.

\section{Availability of data and materials}

The datasets generated and/or analyzed during the present study are not publicly available, but they are available from the corresponding author on reasonable request.

\section{Ethics approval and consent to participate}

Approval of the study and use of the SPACE-cohort was granted by the University Medical Centre Utrecht institutional review board. Requirement for consent was waived because the data was analyzed anonymously. 


\section{Consent for publication}

Not applicable.

\section{Competing interests}

The authors declare that they have no competing interests.

\section{Author details}

'Department of Internal Medicine, division Acute Medicine, University Medical Centre Utrecht, Heidelberglaan 100, 3584, CX, Utrecht, the Netherlands. ${ }^{2}$ Department of Internal Medicine, division Infectious Disease, University Medical Centre Utrecht, Heidelberglaan 100, 3584, CX, Utrecht, the Netherlands.

\section{Received: 11 September 2019 Accepted: 19 November 2019} Published online: 03 December 2019

\section{References}

1. Singer $M$, Deutschman CS, Seymour $C$, et al. The third international consensus definitions for sepsis and septic shock (sepsis-3). JAMA. 2016;315:801-10.

2. Fleischmann C, Scherag A, Adhikari NKJ, Hartog CS, Tsaganos T, Schlattmann P, Angus DC, Reinhart K. Assessment of global incidence and mortality of hospital-treated sepsis current estimates and limitations. Am J Respir Crit Care Med. 2016;193:259-72.

3. Bone RC, Balk RA, Cerra FB, Dellinger RP, Fein AM, Knaus WA, Schein RMH, Sibbald WJ. (1992) definitions for sepsis and organ failure and guidelines for the use of innovative therapies in sepsis. Chest:1644-55.

4. Levy MM, Fink MP, Marshall JC, Abraham E, Angus D, Cook D, Cohen J, Opal SM, Vincent JL, Ramsay G (2003) 2001 SCCM/ESICM/ACCP/ATS/SIS international Sepsis definitions conference. In: Intensive Care Med. pp 530-538.

5. Churpek MM, Zadravecz FJ, Winslow C, Howell MD, Edelson DP. Incidence and prognostic value of the systemic inflammatory response syndrome and organ dysfunctions in ward patients. Am J Respir Crit Care Med. 2015;192: 958-64

6. Kaukonen K-M, Bailey M, Pilcher D, Cooper DJ, Bellomo R. Systemic inflammatory response syndrome criteria in defining severe Sepsis. N Engl J Med. 2015;372:1629-38.

7. van der Woude SW, van Doormaal FF, Hutten BA, Nellen F J, Holleman F. Classifying sepsis patients in the emergency department using SIRS, qSOFA or MEWS. Neth J Med. 2018;76:158-66.

8. Askim A, Moser F, Gustad LT, Stene H, Gundersen M, Asvold BO, Dale J, Bjornsen LP, Damas JK, Solligard E. Poor performance of quick-SOFA (qSOFA) score in predicting severe sepsis and mortality - a prospective study of patients admitted with infection to the emergency department. Scand J Trauma Resusc Emerg Med. 2017:25:56.

9. Churpek MM, Snyder A, Han X, Sokol S, Pettit N, Howell MD, Edelson DP. Quick Sepsis-related organ failure assessment, systemic inflammatory response syndrome, and early warning scores for detecting clinical deterioration in infected patients outside the intensive care unit. Am J Respir Crit Care Med. 2017;195:906-11.

10. de Groot B, Stolwijk F, Warmerdam M, Lucke JA, Singh GK, Abbas M, Mooijaart SP, Ansems A, Esteve Cuevas L, Rijpsma D. The most commonly used disease severity scores are inappropriate for risk stratification of older emergency department sepsis patients: an observational multi-Centre study. Scand J Trauma Resusc Emerg Med. 2017;25:91.

11. Tonelli M, Sacks F, Arnold M, Moye L, Davis B, Pfeffer M. Relation between red blood cell distribution width and cardiovascular event rate in people with coronary disease. Circulation. 2008;117:163-8.

12. Ani C, Ovbiagele B. Elevated red blood cell distribution width predicts mortality in persons with known stroke. J Neurol Sci. 2009:277:103-8.

13. C.V. H, A.K. M, T. T, M. G-M, S.J. S. Usefulness of red cell distribution width as a prognostic marker in pulmonary hypertension. Am J Cardiol. 2009;104:868-72.

14. Sangoi MB, Da Silva SH, Paz Da Silva JE, Moresco RN. Relation between red blood cell distribution width and mortality after acute myocardial infarction. Int J Cardiol. 2011;146:278-80.

15. Oh HJ, Park JT, Kim JK, Yoo DE, Kim SJ, Han SH, Kang SW, Choi KH, Yoo TH Red blood cell distribution width is an independent predictor of mortality in acute kidney injury patients treated with continuous renal replacement therapy. Nephrol Dial Transplant. 2012;27:589-94.

16. Seyhan EC, Özgül MA, Tutar N, Ömür I, Uysal A, Altin S. Red blood cell distribution and survival in patients with chronic obstructive pulmonary disease. COPD J Chronic Obstr Pulm Dis. 2013;10:416-24.
17. Bilal A, Farooq JH, Kiani I, Assad S, Ghazanfar H, Ahmed I. Importance of mean red cell distribution width in hypertensive patients. Cureus. 2016. https://doi.org/10.7759/cureus.902.

18. Guray Y, Ipek EG, Guray U, Demirkan B, Kafes H, Asarcikli LD, Cabuk G, Yilmaz MB. Red cell distribution width predicts mortality in infective endocarditis. Arch Cardiovasc Dis. 2014;107:299-307.

19. Bello S, Fandos S, Lasierra AB, Mincholé E, Panadero C, Simon AL, Gavin O, De Pablo F, Menendez R, Torres A. Red blood cell distribution width [RDW] and long-term mortality after community-acquired pneumonia. A comparison with proadrenomedullin. Respir Med. 2015;109:1193-206.

20. Braun E, Kheir J, Mashiach T, Naffaa M, Azzam ZS. Is elevated red cell distribution width a prognostic predictor in adult patients with community acquired pneumonia? BMC Infect Dis. 2014. https://doi.org/10.1186/1471-2334-14-129.

21. Lorente L, Martín MM, Abreu-González P, et al. Red blood cell distribution width during the first week is associated with severity and mortality in septic patients. PLOS One. 2014. https://doi.org/10.1371/journal.pone.0105436.

22. Sadaka F, O'Brien J, Prakash S. Red cell distribution width and outcome in patients with septic shock. J Intensive Care Med. 2013;28:307-13.

23. Jo YH, Kim K, Lee JH, Kang C, Kim T, Park HM, Kang KW, Kim J, Rhee JE. Red cell distribution width is a prognostic factor in severe sepsis and septic shock. Am J Emerg Med. 2013;31:545-8.

24. Kim S, Lee K, Kim I, Jung S, Kim M-J. Red cell distribution width and early mortality in elderly patients with severe sepsis and septic shock. Clin Exp Emerg Med. 2015;2:155-61.

25. Wang AY, Ma HP, Kao WF, Tsai SH, Chang CK. Red blood cell distribution width is associated with mortality in elderly patients with sepsis. Am J Emerg Med. 2018;36:949-53.

26. Varma S, Sharma N, Varma N, Bhalla A, Jandial A, Kumar S. Elevated red cell distribution width as a prognostic marker in severe sepsis: a prospective observational study. Indian J Crit Care Med. 2017;21:552-62.

27. Patel KV, Ferrucci L, Ershler WB, Longo DL, Gurainik JM. Red blood cell distribution width and the risk of death in middle-aged and older adults. Arch Intern Med. 2009;169:515-23.

28. Douglas SW, Adamson JW. The anemia of chronic disorders: studies of marrow regulation and iron metabolism. Blood. 1975;45:55-65.

29. Ernst Bernhard Jelkmann W, Ann Liebert M, Jelkmann W. Proinflammatory cytokines lowering erythropoietin production Thrombopoietin regulation view project regenerative medicine view project Proinflammatory cytokines lowering erythropoietin production. Artic J Interf Cytokine Res. 1998;18:555-9.

30. Friedman JS, Lopez MF, Fleming MD, Rivera A, Martin FM, Welsh ML, Boyd A, Doctrow SR, Burakoff SJ. SOD2-deficiency anemia: protein oxidation and altered protein expression reveal targets of damage, stress response, and antioxidant responsiveness. Blood. 2004;104:2565-73.

31. Pierce CN, Larson DF. Inflammatory cytokine inhibition of erythropoiesis in patients implanted with a mechanical circulatory assist device. Perfusion. 2005;20:83-90.

32. Weiss G, Goodnough LT. Anemia of chronic disease. N Engl J Med. 2005; 35210352:1011-23.

33. Kolls JK. Oxidative stress in sepsis: a redox redux. J Clin Invest. 2006;116:860-3.

34. Ghaffari S. Oxidative stress in the regulation of Normal and neoplastic hematopoiesis. Antioxid Redox Signal. 2008;10:1923-40.

35. Semba RD, Patel KV, Ferrucci L, Sun K, Roy CN, Guralnik JM, Fried LP. Serum antioxidants and inflammation predict red cell distribution width in older women: the Women's health and aging study I. Clin Nutr. 2010;29:600-4.

36. Lippi G, Targher G, Montagnana M, Salvagno GL, Zoppini G, Guidi GC. Relation between red blood cell distribution width and inflammatory biomarkers in a large cohort of unselected outpatients. Arch Pathol Lab Med. 2009;133:628-32.

37. Armstrong $B$, Walthall $H$, Clancy $M$, Mullee $M$, Simpson $H$. Recording of vital signs in a district general hospital emergency department. Emerg Med J. 2008;25:799-802.

\section{Publisher's Note}

Springer Nature remains neutral with regard to jurisdictional claims in published maps and institutional affiliations. 